\title{
Preparation and characterization of an ultrahydrophobic surface based on a stearic acid self-assembled monolayer over polyethyleneimine thin films
}

\author{
Sili Ren ${ }^{\text {a,b,c,d }}$, Shengrong Yang ${ }^{\mathrm{b}}$, Yapu Zhao ${ }^{\mathrm{c}}$, Tongxi Yu ${ }^{\mathrm{d}}$, Xudong Xiao ${ }^{\mathrm{a}, *}$ \\ a Department of Physics and Institute of Nano Science and Technology, The Hong Kong University of Science and Technology, \\ Hong Kong, China \\ b State key Laboratory of Solid Lubrication, Lanzhou Institute of Chemical Physics, Chinese Academy of Sciences, \\ Lanzhou 730000, China \\ ' State key Laboratory of Nonlinear Mechanics, Institute of Mechanics, Chinese Academy of Sciences, Beijing 100080, China \\ ${ }^{\mathrm{d}}$ Department of Mechanical Engineering, The Hong Kong University of Science and Technology, Hong Kong, China
}

Received 7 May 2003; accepted for publication 21 September 2003

\begin{abstract}
A novel ultrahydrophobic ultrathin film was prepared by stearic acid (STA) chemically adsorbed onto the polyethyleneimine (PEI) coated aluminum wafer. The formation and the structure of the films have been characterized by means of water contact angle measurement, ellipsometry, Fourier transformation infrared spectroscopy, and X-ray photoelectron spectroscopy. The static contact angle for water on the surface of this ultrathin organic film was measured to be as high as $166^{\circ}$. Apart from the hydrophobic STA monolayer, the needle-like surface nanostructures with enough roughness was found to be essential for the generation of ultrahydrophobicity. We suggest that a composite interface formed by the needle-like surface nanostructures, water droplet, and air trapped in the crevices is responsible for the superior water-repellent property.
\end{abstract}

(c) 2003 Elsevier B.V. All rights reserved.

Keywords: Self-assembly; Surface structure, morphology, roughness, and topography; Aluminum; Ellipsometry; Photoelectron spectroscopy; Interface states

\section{Introduction}

Wettability is an important property of solid surfaces from both theoretical and technological points of view [1-5]. For example, in the fast developing micro-electro-mechanical systems

\footnotetext{
${ }^{*}$ Corresponding author. Tel.: +852-2358-7494; fax: 8522358-1652.

E-mail address: phxudong@ust.hk (X. Xiao).
}

(MEMS), which are known for their potential wide applications and low unit cost [6], the large surface-area to volume ratios raise serious adhesive and frictional problems for their operations. With typical surface separations in the range of 500-2000 nm, water droplets can be easily trapped between the high surface tension micromachined structures and produce strong capillary forces [7]. In order to alleviate these adhesive related problems, both the topography and the chemical composition of the contacting surfaces must be 
controlled in order to reduce the surface hydrophilicity.

Recently, much attention has been attracted to surfaces with ultrahydrophobic properties $[1,2,8-$ 14] (Hydrophobic surfaces with water contact angles above $\sim 150^{\circ}$ are called "ultrahydrophobic" [8]). Chen et al. [1] reported that the plasma polymerized heptafluorobutylacrylate (HFBA) thin layer on smooth polyethyleneterephthalate (PET) film possessed advancing and receding water contact angles of $174^{\circ}$ and $173^{\circ}$, respectively. A transparent ultrahydrophobic film on Pyrex glass plates, prepared by sublimation of metal acetylacetonate and a subsequent coating of fluoroalkylsilane, was found to have contact and sliding angles about $160.9^{\circ}$ and $1^{\circ}$, respectively [2,14]. Onda et al. [9] prepared a fractal surface by dipping a glass plate into melted alkylketene dimmer (AKD) and achieved a contact angle about $174^{\circ}$. The porous surface formed on aligned carbon nanotubes, prepared by pyrolysis of metal phthalocyanines and then modified with fluoroalkylsilane, was found to have a water contact angle about $172^{\circ}$ [11]. Tadanaga et al. [13] reported that alumina gel films on soda lime glass plates modified with fluoroalkyltrimethoxysilane could give a water contact angle about $165^{\circ}$. All these examples have a common feature that they were obtained through a combination of surface roughness and hydrophobic materials. In other words, both the surface geometrical structure and the chemical composition control the wettability of the solid surfaces.

In this paper, we report the formation and characterization of an ultrahydrophobic selfassembled stearic acid (STA) monolayer formed on an aluminum substrate coated with an organic polymer film. Compared to the ultrahydrophobic systems mentioned above, our preparation method is even simpler. Branched polyethyleneimine $\{\mathrm{PEI}$, $\left.-\left[\mathrm{C}_{2} \mathrm{H}_{5} \mathrm{NHC}_{2} \mathrm{H}_{5} \mathrm{~N}\left(\mathrm{C}_{2} \mathrm{H}_{5} \mathrm{NH}_{2}\right) \mathrm{C}_{2} \mathrm{H}_{5} \mathrm{NH}\right]_{n}-\right\}$, with a distribution of primary, secondary, and tertiary amine groups in the ratio of $1: 2: 1$, can be easily adsorbed onto any hydroxylated solid surfaces through hydrogen bonds and van der Waals forces [15-17]. The primary and secondary amine groups can be readily modified by phosgene, thiophosgene, cyanuric chloride and glutaraldehyde [15]. In our study, we first form a thin layer of PEI on a hydroxylated aluminum surface and subsequently grow a self-assembled STA monolayer through the reaction between the carboxylic group in STA molecules and the amine groups in PEI. Such an organic film was found to have a water contact angle as large as $166^{\circ}$. While part of the results was reported in a conference [18], in this paper we will present the detailed study of the formation and characterization of this ultrahydrophobic film. We will particularly focus on the preparation conditions and the structures of stable ultrahydrophobic films, and systematically study the relationship between the surface morphology and the contact angles.

\section{Experimental section}

\subsection{Materials}

Polyethyleneimine (PEI, MW = 50,000-60,000), $50 \mathrm{wt} \%$ solution in water, was obtained from ACROS (New Jersey, USA). Stearic aicd (STA) and $N, N^{\prime}$-dicyclohexylcarbodiimide (DCCD) (Sheshan Chemical, Shanghai, China) were of analytical purity and used after purification. The solvent $n$-hexane (purity $>98 \%$ ) was used as received.

\subsection{Substrate preparation}

Two types of aluminum substrates, polished aluminum wafer and evaporated aluminum film on glass plates, were used in this study. Before coating the polymer films and the STA monolayers, the substrates were first ultrasonicated sequentially in methanol and acetone each for $2 \mathrm{~min}$ and then boiled in water to roughen the surfaces. To achieve different roughness, the duration of boiling was varied.

\subsection{Preparation of the stearic acid monolayer}

A thin layer of PEI was first formed by immersing the aluminum substrates into a dilute aqueous solution of PEI $(0.2 \mathrm{wt} \%)$. After rinsing with ultrapure water and dried in the $\mathrm{N}_{2}$ flows, the 
PEI-coated aluminum substrates were put into a dilute solution of STA and DCCD mixture in $n$ hexane for certain time (concentration of STA and DCCD are both $3 \mathrm{mM}$ ). DCCD was used here as dehydration regent for the reaction of STA and PEI. At the end, the samples were washed sequentially with $n$-hexane, acetone and ultrapure water in order to get rid of the physisorbed impurities. To investigate the coverage of the films, the immersion time in the above solutions was varied.

\subsection{Contact angle measurements}

The sessile drop method was used for water contact angle measurements with a contact angle goniometer (Model 100-00, Ramé-hart Inc., Mountain Lakes, NJ, USA). Advancing and receding water contact angles were obtained by keeping a capillary pipette of the microsyringe immersed in the water droplet to increase or decrease the volume. The amount of water droplets used for the measurement was about 3-4 $\mu \mathrm{L}$. For all the contact angles, at least five measurements on different sample spots were made for each specimen.

\subsection{AFM measurements}

The surface morphology measurements were carried out with a home-made atomic force microscope (AFM) controlled by RHK electronics (RHK Technology, Rochester Hills, MI, USA) in the contact mode. Commercially available sharpened pyramidal $\mathrm{Si}_{3} \mathrm{~N}_{4}$ tip with a half cone angle of about $18^{\circ}$ and curvature radius of less than $20 \mathrm{~nm}$ (Park Instruments, Sunnyvale, CA, USA) was employed.

\subsection{Infrared Spectroscopy}

Reflection-absorption IR spectrum was measured on a Fourier transformation infrared spectrometer (IFS $66 \mathrm{~V} / \mathrm{S}$, Bruker, Germany) using an incident angle of $83^{\circ}$. The spectrum was collected for 1000 scans at a resolution of $4 \mathrm{~cm}^{-1}$. A freshly evaporated aluminum film on glass plates was used as the reference. In order to eliminate or decrease the interference of $\mathrm{H}_{2} \mathrm{O}$ and $\mathrm{CO}_{2}$, the sample chamber and the optical chamber were both evacuated to 3 mbar.

\subsection{XPS characterization}

The chemical state of the elements in the ultrahydrophobic surface has also been analyzed on a multi-functional X-ray photoelectron spectroscope (PHI-5702, Perkin-Elmer, USA). The Al K $\alpha$ line was used as the excitation source. The binding energy $284.8 \mathrm{eV}$ of $\mathrm{C} 1 \mathrm{~s}$ in hydrocarbon was used as reference.

\subsection{Ellipsometric measurement}

The thickness of the films was measured on an ellipsometer (L116-E, Gaertner, USA). In the measurement, a He-Ne laser beam $(632.8 \mathrm{~nm})$ was incident at an angle of $70^{\circ}$ to the sample. The real and imaginary part of the refractive index of the Al substrate was first measured as 1.15 and -4.9 , respectively. A real refractive index of 1.50 was assumed for all the organic films. Five replicate measurements were carried out for each specimen and the thickness was recorded to an accuracy of $\pm 0.3 \mathrm{~nm}$. In the ellipsometry and infrared spectroscopy measurements, in order to eliminate the influence of the large substrate roughness, smooth Al $(\mathrm{rms} \sim 0.9 \mathrm{~nm}$ ) substrates were used without roughening in boiling water.

\section{Results and discussion}

\subsection{Preparation and structure of the ultrahydro- phobic film}

We first studied the appropriate deposition conditions for producing the ultrahydrophobic films. In Fig. 1, the water contact angles as a function of the adsorbing time for STA and PEI are plotted. For both smooth and roughened (boiled in water for $5 \mathrm{~min}$ ) Al substrates, after immersing in aqueous solution of PEI $(0.2 \mathrm{wt} \%)$ for $15 \mathrm{~min}$, the water contact angle first increases with the immersion time in STA solution (a mixture of STA and DCCD in $n$-hexane, whose 


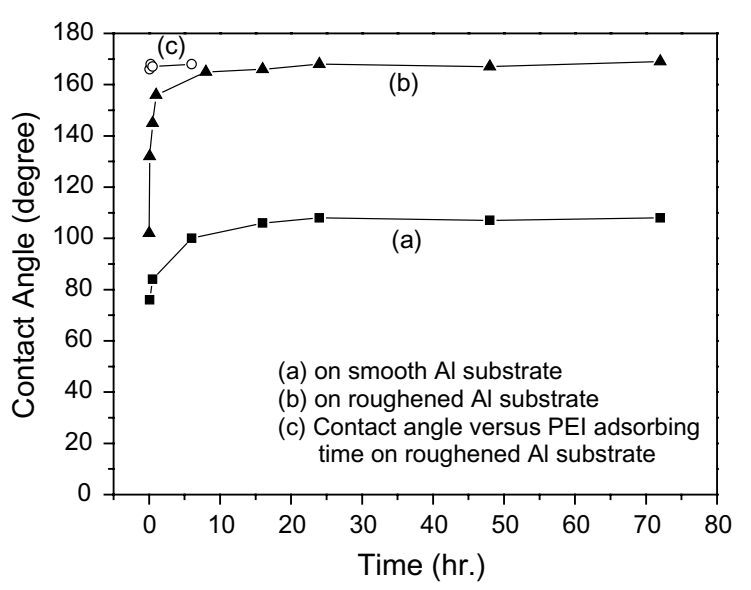

Fig. 1. The water contact angle as a function of immersion time in solution of STA for (a) roughened, (b) flat aluminum substrates after 15 min immersion in a PEI solution and (c) the water contact angle as a function of immersion time in solution of PEI followed with $24 \mathrm{~h}$ immersion in the STA solution.

concentrations are both $3 \mathrm{mM}$ ) and then reaches a saturation value of $105^{\circ}$ and $166^{\circ}$ respectively. The saturation coverage of STA is reached by an immersion time of about $10 \mathrm{~h}$ in STA solution. Further increasing the immersion time no longer results in a larger water contact angle. To investigate the PEI immersion time effect, we vary the immersion time of roughened Al substrates in PEI solution $(0.2 \mathrm{wt} \%)$ from $5 \mathrm{~min}$ to $6 \mathrm{~h}$ with a subsequent $24 \mathrm{~h}$ immersion in the above STA solution. Little difference is observed for the water contact angles. This indicates that the PEI adsorption is a fast process and the extension of the PEI adsorbing time does not produce a better quality STA film. Based on the above results, we will fix the immersion time in PEI for 15 min and in STA for $24 \mathrm{~h}$ respectively for samples used in the rest study.

It is believed that PEI is only physically adsorbed on the Al substrates through van der Waals interaction and hydrogen bond [15-17] since the amine group would not react with $\mathrm{Al}_{2} \mathrm{O}_{3}$. The interaction between STA and PEI is through chemical bonds. In Fig. 2, we present the reflectionabsorption infrared spectrum of the STA film on PEI-coated smooth Al substrate in the frequency range of $3500-1000 \mathrm{~cm}^{-1}$. Both the asymmetric

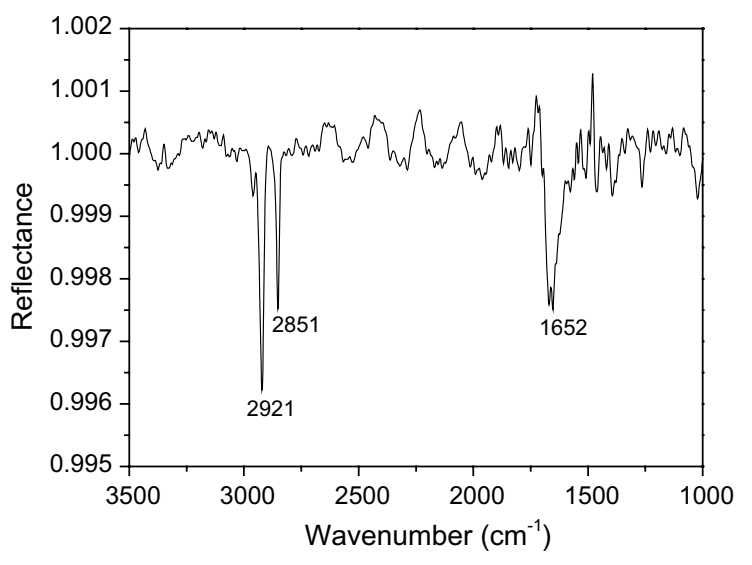

Fig. 2. The reflection-absorption infrared spectrum of the STA monolayer on PEI-coated aluminum surface in the frequency range of $3500-1000 \mathrm{~cm}^{-1}$.

$\left(v_{\text {as }}\left(\mathrm{CH}_{2}\right)\right)$ and symmetric $\left(v_{\mathrm{s}}\left(\mathrm{CH}_{2}\right)\right)$ methylene vibration peaks appear clearly at 2921 and 2851 $\mathrm{cm}^{-1}$, respectively. The peak at $1652 \mathrm{~cm}^{-1}$, arising from amide $\mathrm{I}$ band $(v \mathrm{O}=\mathrm{C})$ [19], is a clear evidence of the chemical reaction between carboxyl group in the STA molecules and the amine groups in PEI, forming $\mathrm{O}=\mathrm{C}-\mathrm{N}-\mathrm{C}$ bond. The peaks for the amide II $\left(v \mathrm{NH}\right.$ at $\left.\sim 1530 \mathrm{~cm}^{-1}\right)$ and amide III bands $\left(v \mathrm{C}-\mathrm{N}\right.$ at $\left.\sim 1300 \mathrm{~cm}^{-1}\right)$ are however too weak to be observed.

In order to further characterize the interaction between STA and PEI, we performed XPS measurement on a sample with ultrahydrophobicity. The results are shown in Fig. 3. The Al $2 p$ peak at $74.2 \mathrm{eV}$ (Fig. 3a) indicates that the $\mathrm{Al}$ element on the substrate surface is in a $\mathrm{Al}_{2} \mathrm{O}_{3}$ or $\mathrm{AlO}(\mathrm{OH})$ state after boiled in water [20,21], consistent with the common knowledge that $\mathrm{Al}$ is oxidized at ambient conditions. The $\mathrm{O} 1 \mathrm{~s}$ peak at $531.6 \mathrm{eV}$ can be assigned to $\mathrm{O}$ elements in $\mathrm{Al}_{2} \mathrm{O}_{3}$ or the carboxyl group (Fig. 3b) [21,22]. There are three peaks arising from $\mathrm{C} 1 \mathrm{~s}$ (Fig. 3c). The first peak at 284.8 $\mathrm{eV}$ can be attributed to the $\mathrm{CH}_{2}$ group in STA, while the second peak at $286.0 \mathrm{eV}$ might originate from the $\mathrm{C}$ atoms bonded to the $\mathrm{N}$ atoms $(\mathrm{O}=\mathrm{C}-$ $\left.\mathrm{N}-\mathrm{C}^{*}\right)$ [16]. The third peak at $287.6 \mathrm{eV}$ comes from the carboxyl $\mathrm{C}$ atom $\left(\mathrm{O}=\mathrm{C}^{*}-\mathrm{N}-\mathrm{C}\right)$ [16]. The above assignments clearly indicate the chemical reaction between STA and PEI. The spectrum for 

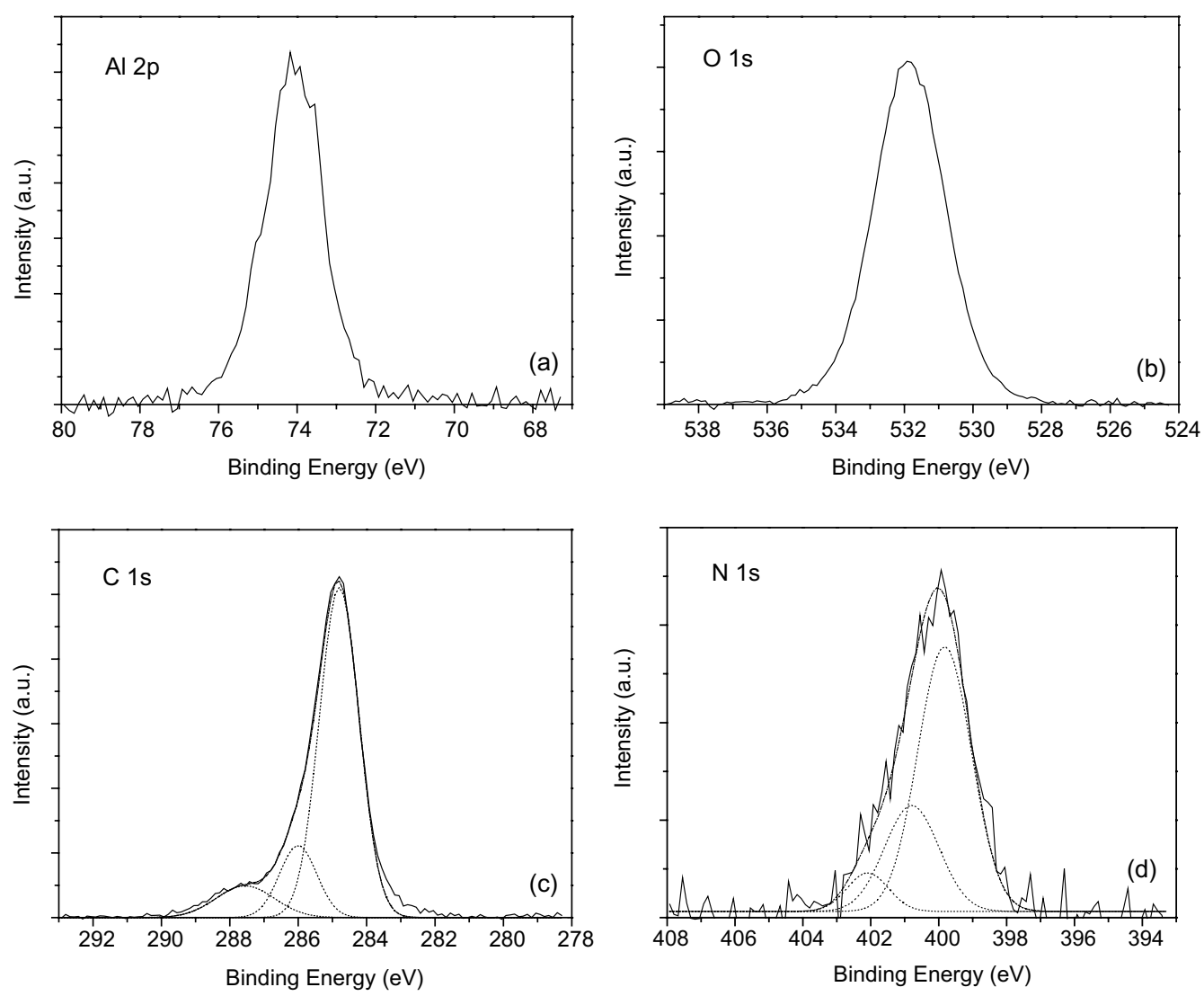

Fig. 3. XPS spectra of the ultrahydrophobic film on a roughened aluminum substrate.

$\mathrm{N} 1 \mathrm{~s}$ spectrum further supports this conclusion (Fig. 3d). While the peak at $399.8 \mathrm{eV}$ is assigned to the $\mathrm{N}$ atoms in the amine group, the peak appeared at $400.8 \mathrm{eV}$ can be attributed to the $\mathrm{N}$ atom bonded to the carbonyl group $\left(\mathrm{O}=\mathrm{C}-\mathrm{N}^{*}\right)[16,23]$. This chemical shift of $\sim 1.0 \mathrm{eV}$ due to the electronwithdrawing effect of the carbonyl group is a little bigger than that of $0.8 \mathrm{eV}$ reported in the literature [16]. As to the peak at $402.0 \mathrm{eV}$, it might come from the oxidation of some amine group in PEI [24].

The formation of chemical bonds between STA and PEI implies that the STA can only be a monolayer thick. In order to confirm this, we measured the film thickness using ellipsometry. To reduce the scattering of light in the measurement, we used a smooth Al substrate without roughening. The thickness of the PEI film after $15 \mathrm{~min}$ immersion is $\sim 5.8 \mathrm{~nm}$ and the STA film after $24 \mathrm{~h}$ immersion is $\sim 2.0 \mathrm{~nm}$. The measured thickness of the STA layer in the present work is somewhat smaller than that of $\sim 2.6 \mathrm{~nm}$ for the STA SAMs on $\mathrm{Al}$ [25] even considering the large uncertainty of $0.3 \mathrm{~nm}$ in our measurement, indicating that the STA monolayer is not very densely packed. This could be attributed to two possible causes: one is that the long-chain STA molecules might be tilted on the PEI surface; the other is that the STA monolayer coverage is incomplete. The latter could be due to the incomplete derivatization of the PEI by STA molecules or a low density of the amine group on the PEI surface.

We have also examined the role of DCCD in the formation of STA monolayer. Table 1 shows the water contact angles on various surfaces studied. The contact angles of water on the roughened 
Table 1

Water contact angles on various surfaces with similar roughness and asperity structures

\begin{tabular}{cllll}
\hline Surface & Aluminum & PEI coating & STA & $\begin{array}{l}\text { mono- } \\
\text { layer }\end{array}$ \\
\hline $\begin{array}{c}\text { Contact } \\
\text { angle }\left(^{\circ}\right)\end{array}$ & $<5$ & $<5$ & 166 & $120^{\mathrm{a}}$ \\
\hline
\end{tabular}

${ }^{a}$ Represents the contact angles on the STA film prepared in the absence of DCCD in the reacting solution.

polished Al surface and PEI coated surface are below $5^{\circ}$, indicating that both rough and PEIcoated Al surfaces are strongly hydrophilic. Once the PEI-coated Al substrate was immersed in the STA and DCCD mixture solution for $24 \mathrm{~h}$, the surface become ultrahydrophobic with a contact angle as high as $166^{\circ}$. The water droplet on this surface is very spherical. The large change of the contact angles reflects the changes of the chemical composition on the surface. However, if the same kind PEI-coated Al substrate is immersed in STA solution without DCCD, the contact angle is only about $120^{\circ}$, indicating that the STA layer onto the PEI surface is of lower quality. Without DCCD, STA might adsorb to the surface only through hydrogen-bonding or electrostatic interaction (Scheme 1). Similar conclusions was also made by
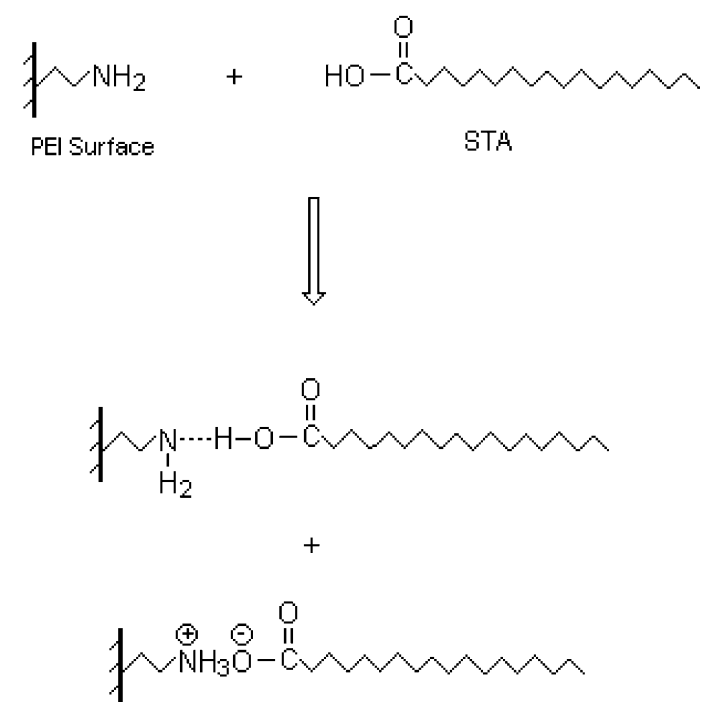

Scheme 1. Adsorption of the STA molecules onto PEI surface in the absence of DCCD in the solution.
Cai et al. [26] in preparing the complex composition of $\mathrm{PEI}(\mathrm{OA})_{x}$ by refluxing the mixed dilute aqueous PEI solution and octadecanoic acid (OA) solution in carbon tetrachloride without DCCD. Their FTIR results indicated that the PEI(OA $)_{x}$ composition was combined through proton transferring or hydrogen bonding. The addition of DCCD as dehydration regent into the STA solution can help the chemical reaction between the amine groups in PEI and carboxyl group in STA molecules to form covalent amide bond [27]. The reaction process is shown as Scheme 2.

\subsection{Mechanism of the ultrahydrophobicity}

\subsubsection{Effects of surface roughness}

It is well known that the water contact angles on smooth hydrophobic surfaces are generally not exceeding $115^{\circ}-120^{\circ}$. For example, the contact angles are about $112^{\circ}$ and $115^{\circ}$ for long chain hydrocarbon and fluorocarbon self-assembled monolayer surfaces, respectively [5]. However, the situation is quite different when the surface is roughened [28]. In order to investigate such effect in our system, we have prepared STA monolayers on PEI coated
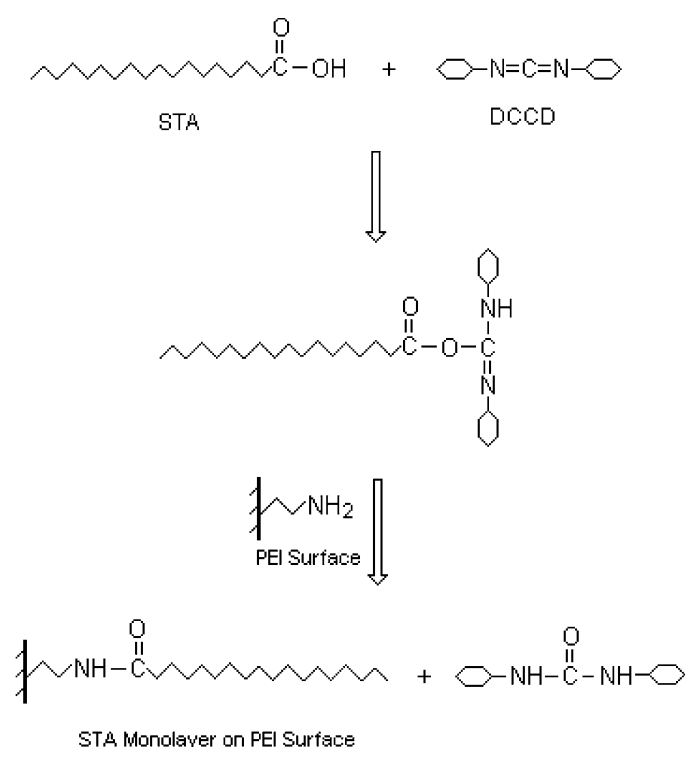

Scheme 2. The generation of an STA monolayer on the PEIcoated aluminum surface by chemical adsorption in the presence of DCCD in the reacting solution as a dehydrating agent. 


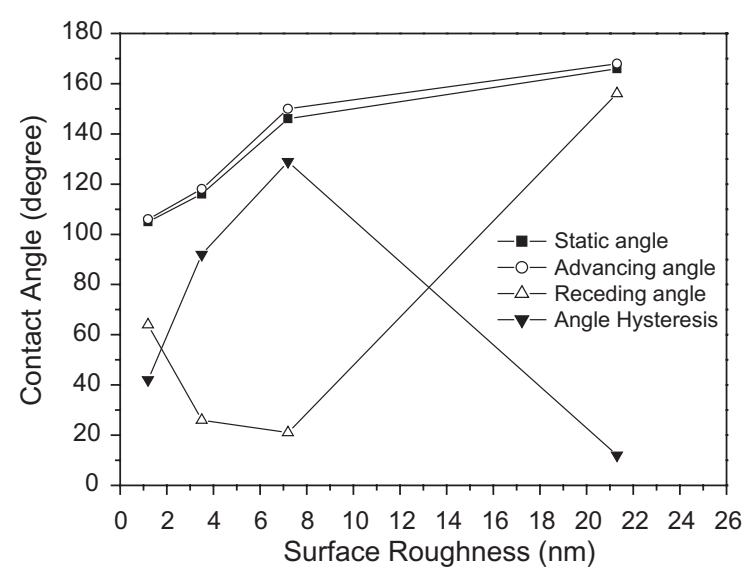

Fig. 4. Relation between the contact angles and the surface roughnesses of the STA monolayers.

aluminum substrates with rms roughness of 1.2 , $3.5,7.2$, and $21.3 \mathrm{~nm}$, respectively. The various surface roughness were controlled by immersing the freshly polished Al substrates in the boiling water for $0,10,60$, and $300 \mathrm{~s}$. Further increasing the boiling time does not result in higher roughness of the substrates. As seen from Fig. 4, the water contact angles on these samples increase with the surface roughness and are $105^{\circ}, 116^{\circ}$, $146^{\circ}$, and $166^{\circ}$, respectively.

The representative AFM images of the surface morphology for the above four samples are shown in Fig. 5. It can be seen that the STA monolayer prepared on the freshly polished Al substrate without having been boiled in water is relative smooth (Fig. 5a). However, once boiled in water for a certain time, the surfaces become rough and develop needle-like structures (Fig. 5b-d). The height of such structures increases with the extension of the boiling time. The roughening process might be attributed to the chemical erosion of water to the freshly polished aluminum surface at high temperature:

$\mathrm{Al}+\mathrm{H}_{2} \mathrm{O} \stackrel{\Delta}{\rightarrow} \mathrm{Al}_{2} \mathrm{O}_{3} \cdot x \mathrm{H}_{2} \mathrm{O}+\mathrm{H}_{2} \uparrow$.

Physical erosion of the air bubble in the boiling water to the surface might also be important. According to Ref. [13], the initial thin $\mathrm{Al}_{2} \mathrm{O}_{3}$ layer on the freshly polished substrates could react with water to form boehmite crystal, $\mathrm{AlO}(\mathrm{OH})$, which

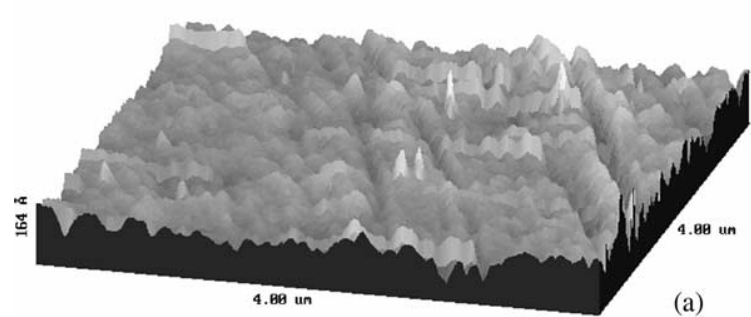

(a)

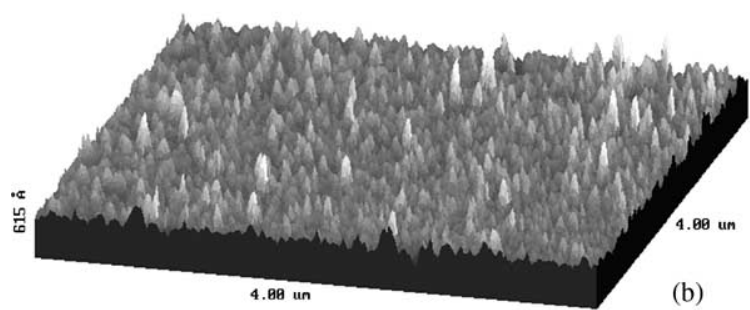

(b)

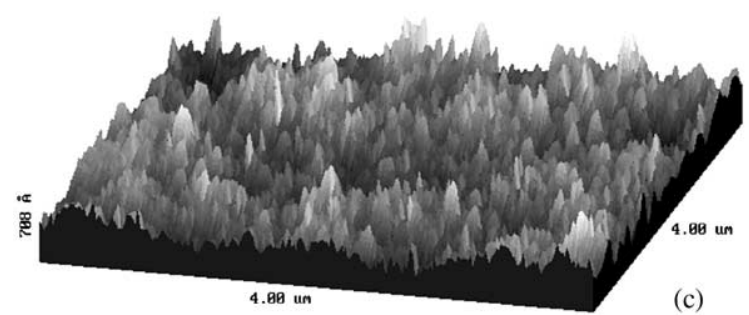

(c)

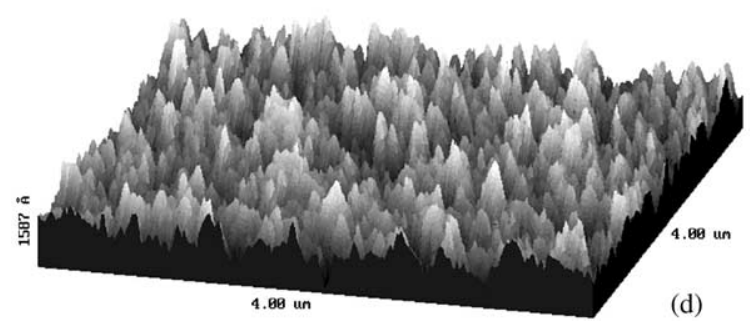

Fig. 5. AFM images of STA monolayers on PEI-coated polished aluminum surface with rms roughness of (a) $\sim 1.2 \mathrm{~nm}$, (b) $3.5 \mathrm{~nm}$, (c) $7.2 \mathrm{~nm}$ and (d) $21.3 \mathrm{~nm}$ respectively.

could dissolve in the boiling water. The correlation between the water contact angle and the surface roughness for the above samples leads us to conclude that large roughness is essential to form the ultrahydrophobic surface. Our findings are similar to those of Miwa et al. [14] who reported that the higher the needle-like peaks the larger the water contact angles. Nakajima et al. [2] also found a similar trend on a transparent ultrahydrophobic thin film prepared by utilizing a sublimation 
material and subsequent coating of a fluoroalkylsilane, except that ultrahydrophobicity does not occur until a much higher roughness. In their study, the water contact angles were found to be about $120^{\circ}$ and $152^{\circ}$ for surfaces with rms roughness of 30 and $90 \mathrm{~nm}$, respectively. This reflects that, besides the roughness, the ultrahydrophobicity must be influenced by other factors such as the surface asperity shapes and the chemical properties of the films.

\subsubsection{Chemical role of $S T A$}

The formation of a STA monolayer is the key to achieve ultrahydrophobicity for the present system. Table 1 shows the water contact angles for aluminum, PEI coating, and STA monolayer, respectively, on substrates boiled in water for $5 \mathrm{~min}$. Clearly, the surfaces of the aluminum and PEIcoated aluminum are hydrophilic. Once with a STA monolayer formed, the static contact angles drastically increased to as high as about $166^{\circ}$.

Fig. 6 shows the AFM images of an aluminum wafer after it has been boiled in water for $5 \mathrm{~min}$ (Fig. 6a), and with a subsequent PEI coating (Fig. $6 \mathrm{~b})$ on top of it. Both of them show needle-like
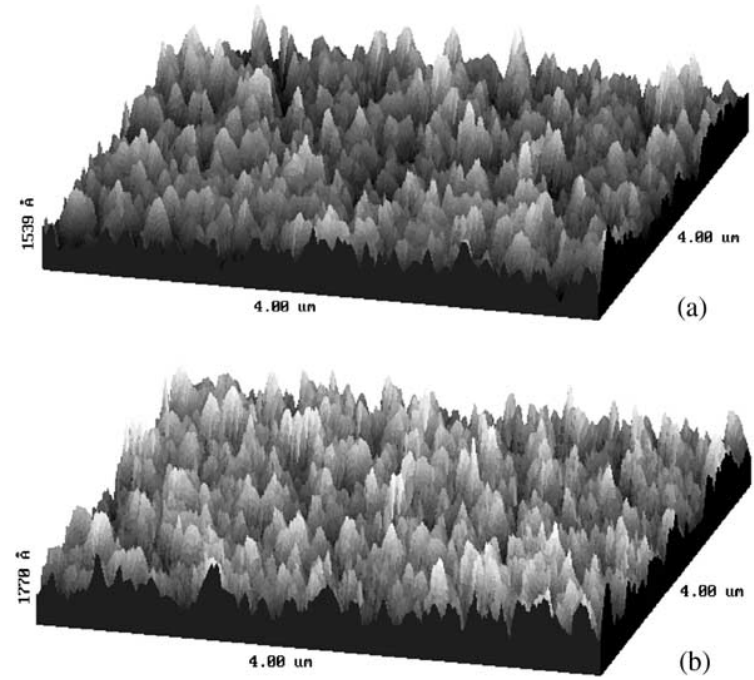

Fig. 6. AFM images of an aluminum wafer after (a) having been boiled in water for 5 min and (b) with subsequent PEI coating on top of it. The rms roughness is 18.5 and $22.0 \mathrm{~nm}$, respectively. structures, similar to that of Fig. 5d. The subsequently grown PEI film and STA monolayer on the water-boiled substrates have little effect on the surface morphology and the roughness of the three surfaces (Figs. 6a, b and 5d) remains more or less the same. With no obvious change in the roughness and structure after the growing of the STA monolayer, the large change in water contact angles clearly demonstrates the chemical role of the STA molecules.

\subsubsection{Effect of asperity shapes}

While it is already clear that both the roughness of the substrate and the STA monolayer are responsible for the ultrahydrophobic behavior, we further show that the asperity shapes also play an important role. We prepared aluminum substrates by thermal evaporation on glass and followed the aforementioned procedure to grow a STA monolayer. By controlling the substrate boiling time in water, a STA monolayer surface with rms roughness of $\sim 16.5 \mathrm{~nm}$ was prepared. Fig. 7(a) shows the surface AFM topography. Unlike the needlelike structure shown in Fig. 5d, two features are present: one is that the shape of the asperities is mushroom-like with somewhat rounded tips; the
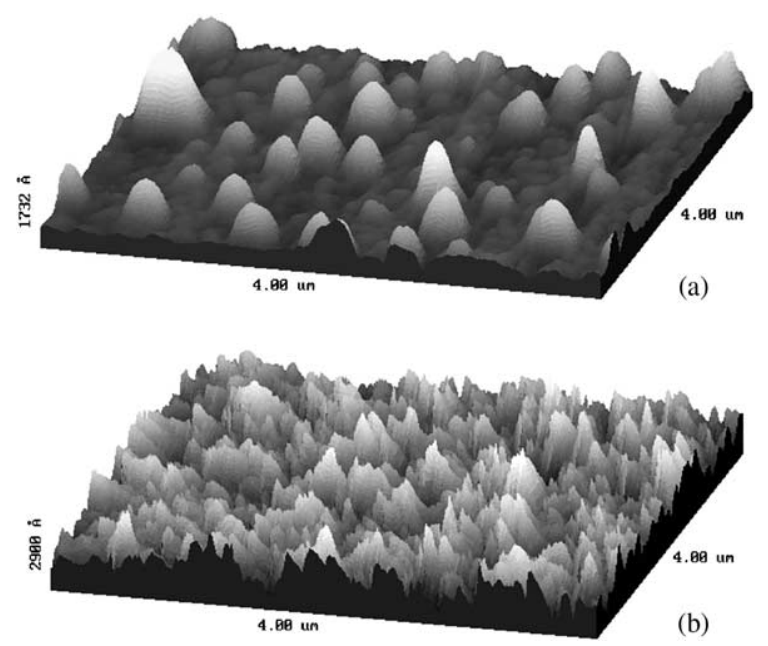

Fig. 7. AFM images of the ultrathin film on evaporated aluminum film with different surface geometric structure and roughness. 
other is that the density of asperities is low. On this sample surface, the water contact angle is only about $118^{\circ}$. Even the rms roughness value is close to that of the sample shown in Fig. 5d, the hydrophobicity is not as strong. This indicates that the roughness value is not the only factor that affects formation of an ultrahydrophobic surface, the structure and the density of the asperities also affect the behavior of the ultrahydrophobicity of a surface. If the water boiling time is further increased to roughen the evaporated aluminum substrate to a rms roughness of $\sim 38.4 \mathrm{~nm}$ and the asperity shape becomes needle-like (Fig. 7b), the prepared STA monolayer surface now becomes water-repellent with a contact angle about $162^{\circ}$. The difference between the saturation roughness of the evaporated aluminum film and polished aluminum wafer after boiled in water might come from the difference in the Al grains and grain boundaries. Despite the different roughness in the two substrates, we found similar water contact angles for the ultrahydrophobic films. This is consistent with what has been reported by Bico et al. [29] that the main parameter in determining the ultrahydrophobicity is the fraction of the liquid actually in contact with the rough solid surface but not the surface roughness itself.

\subsubsection{Contact angles hysteresis}

To better describe the surface wettability, the contact angle hysteresis, i.e. the difference between the advancing and receding contact angles, should be considered as well [1]. This is because a water droplet on a rough surface often resides in a metastable state and exhibits a metastable contact angle $[28,30]$. In this situation, the static contact angle or the advancing contact angle alone is not enough to reflect the wettability of a solid surface. To measure the advancing angle, we gradually increased the water in the droplet with a microsyringe. Similarly, to measure the receding angle, we gradually removed a small amount of liquid from the droplet with a microsyringe. The advancing, receding angles and the hysteresis on the STA surfaces with different roughness are shown in Fig. 4. It is clear that the hysteresis for various rough samples is quite different.

\subsubsection{Mechanism of ultrahydrophobicity}

On a smooth and homogeneous surface, the contact angle $\theta$ for a given liquid droplet is described by Young's equation [31]:

$\gamma_{\mathrm{LA}} \cos \theta=\gamma_{\mathrm{SA}}-\gamma_{\mathrm{SL}}$,

where $\gamma_{\mathrm{LA}}, \gamma_{\mathrm{SA}}$, and $\gamma_{\mathrm{SL}}$ are the interfacial tensions of the liquid-air, solid-air, and solid-liquid interface, respectively. The contact angle $\theta$, also called Yang angle, is intrinsic and unique. However, as calculated in Ref. [30], the relation between free energy and the apparent contact angle for a liquid droplet on a rough surface showed that metastable states existed and were separated by free energy barriers. In other words, different contact angles can be found on rough surfaces. Corresponding to the minimum free energy of the entire system, the equilibrium contact angle $\theta_{\mathrm{w}}$ the Wenzel angle, was given by the Wenzel equation [32]:

$\cos \theta_{\mathrm{w}}=r \cos \theta$,

where $r$ is the ratio of the actual area of a rough surface to the geometric projected area, known as the roughness factor. Since $r$ is always bigger than unity, $\theta_{\mathrm{w}}$ would be increased if $\theta>90^{\circ}$ and decreased if $\theta<90^{\circ}$ by surface roughness. It should be pointed out that it is difficult to measure the Wenzel angle in practice. Experimentally, we can measure the advancing angle, $\theta_{\mathrm{a}}$ and the receding angle, $\theta_{\mathrm{r}}$. For Yang angle $\theta \geqslant 90^{\circ}$, the maximum advancing angle for a noncomposite interface is: $[30,33]$

$\theta_{\mathrm{a}}=\theta+\alpha$

and correspondingly the minimum receding angle is:

$\theta_{\mathrm{r}}=\theta-\alpha$,

where $\alpha$ is the inclination angle of the surface asperities at the contact line.

On the other hand, if a composite interface $[28,34,35]$ is generated by trapping air in the crevices beneath the liquid drop, forming a heterogeneous surface with two phases, air and solid, the equilibrium contact angle $\theta_{\mathrm{c}}$ i.e. the Cassie angle, is then given by the Cassie equation [36]:

$\cos \theta_{\mathrm{c}}=f_{1} \cos \theta_{1}+f_{2} \cos \theta_{2}$, 
where $\theta_{\mathrm{i}}$ and $f_{\mathrm{i}}$ are the Yang angle and the fraction area for each phase beneath the liquid droplet on the heterogeneous surface $\left(f_{2}=1-f_{1}\right)$. As calculated in Ref. [37] and confirmed by experiments, on composite surfaces with enough roughness the height of the energy barriers between metastable states decreases dramatically and the contact angle hysteresis is greatly lowered.

Following the above discussion, our results in Fig. 4 clearly indicate that the composite interface has not yet formed when the surface roughness is low. Only for the sample with $21.3 \mathrm{~nm} \mathrm{rms}$ roughness, a composite interface is formed. These results are qualitatively consistent with the theoretical predictions [37]. As the surface roughness increases from 1.2 to 3.5 and $7.2 \mathrm{~nm}$, the inclination angle $\alpha$ increases. Following Eq. (3), the contact angle hysteresis is expected to increase for noncomposite interfaces. This is consistent with the observed contact angle hysteresis increase from $42^{\circ}$ to $92^{\circ}$ and $129^{\circ}$ respectively. Upon the formation of a composite interface, the contact angle hysteresis is expected to greatly reduce [37], as indeed seen for the sample with $21.3 \mathrm{~nm} \mathrm{rms}$ roughness (a hysteresis of only $12^{\circ}$ ). Using $\theta_{\mathrm{c}} \sim$ $166^{\circ}$ from the experimental results, $\theta_{1} \sim 105^{\circ}$ from the relative smooth sample of Fig. 5a and $\theta_{2} \sim 180^{\circ}$ for air, we obtain $f_{2} \sim 0.96$ from Eq. (4) for the water/air fractional contact area. (It should be pointed out that using the static contact angle of $166^{\circ}$ as the equilibrium contact angle for the ultrahydrophobic surface brings only a minor error for the value of $f_{2}$ ) [1]. This indicates that at the composite interface, water is mostly in contact with air and thus the roughened surface with STA monolayer becomes extremely water-repellent. We also notice that other than the roughness effect, the lower receding angles $\left(64^{\circ}, 26^{\circ}\right.$ and $21^{\circ}$, respectively, for the first three samples in Fig. 4) might reflect that the sample surface was chemically heterogeneous, e.g., the STA monolayer have not completely covered the entire surface. Since the PEI coating are strongly hydrophilic (Table 1), the water contact line would be pinned at the hydrophilic regions and result in a smaller receding contact angle. It has been concluded by others that a small amount of hydrophilic materials on a hydrophobic surface can greatly reduce the receding contact angle, but have little effect on the advancing contact angle [28].

For the STA surfaces with mushroom-like asperities, following the above argument, the composite interface has not formed when the roughness is $16.5 \mathrm{~nm}$. Further increasing the roughness (38.3 $\mathrm{nm})$, so the surface morphology becomes needlelike, the composite interface is formed and gives a large contact angle. For the rough aluminum and PEI-coated aluminum surfaces, because of the hydrophilicity of the materials at the surfaces, water can easily penetrate into the crevices and no air can be trapped at the interface. Thus, the water contact angle remains very low despite of the large roughness of the needle-like surfaces. This analysis shows that the needle-like structure with sufficient roughness and the hydrophobic materials are essential for the generation of the ultrahydrophobic surface.

\section{Conclusion}

We have prepared a novel super water-repellent organic ultrathin film with a water contact angle of $166^{\circ}$ by stearic acid chemically adsorbed onto the polyethyleneimine coated aluminum wafer. The structure and the formation of the film have been characterized by means of contact angle measurement, ellipsometry, Fourier transformation infrared spectroscopy, and X-ray photoelectron spectroscopy. The results show that, by forming covalent amide bond between the carboxyl group in STA molecules and the amine groups in PEI, the STA monolayer is generated on the PEI-coated aluminum surface. Results of the relation between the ultrahydrophobicity and surface nanostructures show that both the rough needle-like geometric nanostructures and hydrophobic materials are essential to the formation of the ultrahydrophobic surface. On such a surface, a composite interface would be formed in the contact area between the nanostructures, the water droplet and the air trapped in the crevices. This composite interface has been attributed to be responsible for the super water-repellent properties. We have also found that the ultrahydrophobic surface has a weak contact angle hysteresis with the advancing 
and receding contact angle about $168^{\circ}$ and $156^{\circ}$, respectively. Our findings on the relation between the nanostructures and the ultrahydrophobicity are instructive to reduce the stiction problems in MEMS. While the roughness of the needle-like structures is expected to increase friction and to facilitate the wear process in a close contact, their effect on the operation of MEMS remains to be investigated since there the typical surface separation is in the range of 500-2000 nm during operation. We are currently working on the roughening process of Si surface to produce needle-like nanostructures in order to eventually apply the present knowledge for the MEMS industry.

\section{Acknowledgements}

The authors wish to acknowledge the financial support from the Hong Kong Research Grant Council through project HKUST6155/00P and from National Natural Science Foundation of China (Grant No. 50275142) and from the Chinese Academy of Sciences (Grant No. KJCX-SW-L2). The research project is also supported by the Joint Lab on Microsystems between the Hong Kong University of Science and Technology (HKUST) and the Institute of Mechanics, CAS, China, under a grant from HKUST (Project No.CMI00/ 01.EG02).

\section{References}

[1] W. Chen, A.Y. Fadeev, M.C. Hsieh, D. Öner, Langmuir 15 (1999) 3395.

[2] A. Nakajima, K. Hashimoto, T. Watanabe, K. Takai, G. Yamauchi, A. Fujishima, Langmuir 16 (2000) 7044.

[3] S.Y. Yang, G.J. Hirasaki, S. Basu, R. Vaidya, J. Petrol. Sci. Eng. 24 (1999) 63.

[4] Y.N. Xia, D. Qin, Y.D. Yin, Curr. Opin. Interface Sci. 6 (2001) 54.

[5] D.Y. Kwok, A.W. Neumann, Adv. Collide Interface Sci. 81 (1999) 167.

[6] S.M. Spearing, Acta. Mater. 48 (2000) 179.

[7] Z. Rymuza, Microsyst. Technol. 5 (1999) 173.

[8] A. Nakajima, A. Fujishima, Adv. Mater. 11 (1999) 1365.

[9] T. Onda, S. Shibuichi, N. Satoh, K. Tsujii, Langmuir 12 (1996) 2125.
[10] K. Tadanaga, N. Katata, T. Minami, J. Am. Ceram. Soc. 80 (1997) 1040.

[11] H.-J. Li, X.-B. Wang, Y.-L. Song, Y.-Q. Liu, Q.-S. Li, L. Jiang, D.-B. Zhu, Chem. J. Chinese Univ. 22 (2001) 759 (in Chinese).

[12] K. Tadanaga, J. Morinaga, A. Matsuda, T. Minami, Chem. Mater. 12 (2000) 590.

[13] K. Tadanaga, N. Katata, T. Minami, J. Am. Ceram. Soc. 80 (1997) 3213.

[14] M. Miwa, A. Nakajima, A. Fujishima, K. Hashimoto, T. Watanabe, Langmuir 16 (2000) 5754.

[15] R. Bahulekar, N.R. Ayyangar, S. Ponrathnam, Enzyme Microb. Technol. 13 (1991) 858.

[16] J.J. Chance, W.C. Purdy, Langmuir 13 (1997) 4487.

[17] S.-L. Ren, S.-R. Yang, Q.-J. Xue, Acta Physico-Chim. Sinica 17 (2001) 97 (in Chinese).

[18] S.-L. Ren, S.-R. Yang, Y.-P. Zhao, X.-D. Xiao, T.-X. Yu, in: Proceedings of the International Conference on Micro \& Nano Systems 2002, International Journal of Nonlinear Sciences and Numerical Simulation 3 (2002) 785 (Special issue).

[19] K. Nakanishi, P.H. Solomon, Infrared Absorption Spectroscopy, 2nd ed., Holden-day Inc press, San Francisco, 1977, p. 42.

[20] E. Paparazzo, Appl. Surf. Sci. 25 (1986) 1.

[21] C.D. Wagner, D.E. Passoja, H.F. Hillery, T.G. KinisKy, H.A. Six, W.T. Jansen, J.A. Taylor, J. Vac. Sci. Technol. 21 (1982) 933.

[22] J. Peeling, F.E. Hruska, D.M. Mckinnon, M.S. Chauhan, N.S. Mclntyre, Can. J. Chem. 56 (1978) 2407.

[23] J. Peeling, F.E. Hruska, N.S. Mclntyre, Can. J. Chem. 56 (1978) 1555.

[24] M. Seno, S. Tsuchiya, J. Electron Spectrosc. Relat. Phenom. 8 (1976) 165.

[25] Y.T. Tao, J. Am. Chem. Soc. 115 (1993) 4350.

[26] Y.L. Cai, D.J. Wang, X.B. Hu, Y.H. Xu, Y. Zhao, J. Wu, D.F. Xu, Macromol. Chem. Phys. 202 (2001) 2434.

[27] D.A. Hutt, G.J. Leggett, Langmuir 13 (1997) 2740.

[28] S.H. Wu, Polymer Interface and Adhesion, Marcel Dekker Press, New York, 1982.

[29] J. Bico, C. Marzolin, D. Quéré, Europhys. Lett. 47 (1999) 220.

[30] J.D. Eick, R.J. Good, A.W. Neumann, J. Colloid Interface Sci. 53 (1975) 235.

[31] T. Young, in: D. Peacock (Ed.), Proc. Roy. Soc., Royal Society of London, London, 1804.

[32] R.N. Wenzel, Ind. Eng. Chem. 28 (1936) 988.

[33] R. Shuttleworth, G.L. Bailey, Discuss. Faraday Soc. 3 (1948) 16.

[34] F.E. Bartell, J.W. Shepard, J. Phys. Chem. 57 (1953) 211.

[35] A.M. Schwartz, F.W. Minor, J. Colloid Sci. 14 (1959) 584.

[36] A.B.D. Cassie, Discuss. Faraday Soc. 3 (1948) 11.

[37] R.E. Johnson Jr., R.H. Dettre, Contact Angle, Wettability, and Adhesion in: F.M. Fowkes (Ed.), Advances in Chemistry 43, American Chemical Society, Washington, DC, 1964, p. 112. 